


\title{
TRANSIENT RESPONSE OF A GENERAL ANISOTROPIC SOLID TO DISLOCATION GROWTH: ALTERNATIVE FORMULATION
}

\author{
LOUIS MiLTON BROCK
}

\begin{abstract}
The response of an unbounded homogeneous general anisotropic solid to dislocation growth can be obtained from a convolution of the body force equivalent of the dislocation with the Green's function. This work considers the transient case of a surface of discontinuity in displacement that expands with time from a point source. The expansion rate is subsonic, but largely arbitrary otherwise. The surface need not be planar, and if so, need not lie in a principal material plane. Some differences in procedure from related studies are noted. The solution expressions use a dislocation description in terms of surface geometry, and have a hybrid but somewhat more explicit form.
\end{abstract}

\section{Introduction}

The radiation field from a spreading dislocation is known [Nabarro 1951; Knopoff and Gilbert 1960] to be obtainable in terms of solutions to the fundamental problems of point forces and couples and their line load counterparts. Such loads can be generalized as the transient body-force equivalent for a dislocation distribution on an internal surface [Burridge and Knopoff 1964]. The equivalent is valid for the inhomogeneous, anisotropic solid, and surface and distribution are (largely) arbitrary.

This article gives an exact transient solution for the growth of a dislocation distribution in a homogeneous, general anisotropic and unbounded solid in terms of the Burridge-Knopoff equivalent. The dislocation spreads at a subsonic (possibly nonuniform) rate from a point source. The dislocation distribution is single valued and remains finite for finite time after its appearance. This problem is not new, but the present study includes some alternative features.

In [Burridge and Knopoff 1964] the dislocation field is defined in terms of the components of a prescribed displacement discontinuity in the principal material basis. For insight into the roles of climb and glide mechanisms, two descriptions of the displacement discontinuity vector are treated here. The first resolves the prescribed displacement discontinuity vector into components normal to, and in the plane of, the surface. Coefficient arrays $\left(d_{i k}^{N S}, d_{i k}^{N N}\right)$ arise, and a third, $d_{i k}^{S S}$, can be defined. Subscripts $(i, k)$ refer to Cartesian coordinates $\left(x_{1}, x_{2}, x_{3}\right)$ in the principal material basis, and $(N, S)$ refer to coordinates normal to and in the plane of the discontinuity surface. The arrays are symmetric in $(N, S)$, and the six elements of each array are symmetric in $(i, k)$. The form of $d_{i k}^{N S}$ resembles the constitutive equation for the general anisotropic solid.

The second description treated is local, that is, it is the components of the displacement discontinuity vector with respect to surface geometry that are prescribed. Three coefficient arrays $\left(d_{i k}^{N T}, d_{i k}^{N S}, d_{i k}^{N N}\right)$ arise, and arrays $\left(d_{i k}^{T T}, d_{i k}^{S S}, d_{i k}^{T S}\right)$ are also defined. Here $N$ refers to the surface normal and $(T, S)$ are

Keywords: transient 3D, dislocation, exact solution, general anisotropy, wave speeds. 
two perpendicular directions in the surface plane. Use of this description in the transient solutions is demonstrated for a planar discontinuity surface, for which coefficient arrays are constant.

The analysis is similar to that for the anisotropic Green's function. That problem is also not new, nor is the use of integral transforms [Willis 1980; Payton 1983; Wang and Achenbach 1994; 1995; Ting and Lee 1997]. Here unilateral temporal and bilateral spatial Laplace transforms [van der Pol and Bremmer 1950; Sneddon 1972] are employed. The spatial transforms are with respect to the Cartesian principal basis, but quasispherical coordinates are introduced in the inversion process. This facilitates the residue calculation process common in anisotropic Green's function study in, for example, [Payton 1983; Wang and Achenbach 1994; 1995; Ting and Lee 1997]. Moreover, the displacements take a hybrid form: their components and coordinates are Cartesian, but the expressions are integrals of real-valued functions in a unit quarter-sphere. Although lacking the simplicity of integration based on the hypercircle or unit sphere [Synge 1957; Wang and Achenbach 1995], the integration is explicitly in terms of polar and azimuthal angles defined in the fixed principal basis. For the uniform dislocation distribution, in fact, integration can be transformed to the contour of the dislocation surface; see [Brock 1986]. Analytical expressions for the poles in the residue calculation - and therefore the three anisotropic wave speeds are also presented; see [Wang and Achenbach 1995].

Finally, it is noted that some well-known formalisms for anisotropy, for example, [Stroh 1958; 1962; Barnett and Lothe 1973; Ting 1996; Ting and Lee 1997], are not invoked here. However, analogous formulas arise in the course of analysis. Solution expression development is the focus here but, for illustration, sample wave speed calculations are given for a transversely isotropic graphite-epoxy solid.

\section{Governing equations}

A Cartesian basis $\boldsymbol{x}\left(x_{1}, x_{2}, x_{3}\right)$ defines the principal material axes for an unbounded, homogeneous linear anisotropic solid. In contracted notation, the stress and strain measures $\left(\sigma_{k}, \epsilon_{k}\right)$ for the solid are related by

$$
\sigma_{k}=C_{k l} \epsilon_{l}, \quad C_{k l}=C_{l k} .
$$

Here $(k, l)$ take on values $(1,2,3,4,5,6)$ and the 21 elasticity parameters $C_{k l}$ are constants. These measures correspond to those in the Cartesian basis as follows: For $k=(1,2,3)$

$$
\sigma_{k}=\sigma_{k k}, \quad \epsilon_{k}=\partial_{k} u_{k} .
$$

The range $k=(4,5,6)$ corresponds to Cartesian shear stresses and strains:

$$
\begin{array}{ll}
\sigma_{4}=\sigma_{23}=\sigma_{32}, & \epsilon_{4}=\partial_{2} u_{3}+\partial_{3} u_{2}, \\
\sigma_{5}=\sigma_{31}=\sigma_{13}, & \epsilon_{5}=\partial_{3} u_{1}+\partial_{1} u_{3}, \\
\sigma_{6}=\sigma_{12}=\sigma_{21}, & \epsilon_{6}=\partial_{1} u_{2}+\partial_{2} u_{1} .
\end{array}
$$

Equation (1) is associated with positive strain energy density when $\epsilon_{k}^{T} C_{k l} \epsilon_{l}>0$. Because $C_{k l}$ constitutes a symmetric $6 \times 6$ matrix with real elements, it can be shown [Hohn 1964; Ting 1996] that the inequality is satisfied when the leading principal minors of $C_{k l}$ are positive, that is, 


$$
\left|\begin{array}{cccc}
C_{11} & C_{12} & \cdots & C_{1 n} \\
C_{12} & C_{22} & \cdots & C_{2 n} \\
\vdots & \vdots & \ddots & \vdots \\
C_{1 n} & C_{2 n} & \cdots & C_{n n}
\end{array}\right|>0 \quad(n \leq 6)
$$

In (1)-(3) $u_{k}$ are the components of $\boldsymbol{u}$ in the $x_{k}$-direction, and $\partial_{k}$ signifies differentiation with respect to $x_{k}$. It is convenient to introduce reference shear modulus and rotational wave speed $\left(\mu, v_{S}\right)$, where

$$
\mu=\max \left(C_{44}, C_{55}, C_{66}\right), \quad v_{S}=\sqrt{\frac{\mu}{\rho}} .
$$

Here $\rho$ is the mass density, and use of (5) gives the time-dependent length measure $\tau=v_{S} \times$ (time). Therefore $u_{k}=u_{k}(\boldsymbol{x}, \tau)$ and $\sigma_{k l}=\sigma_{k l}(\boldsymbol{x}, \tau)$ in (2) and (3), and for $\tau<0$ the unbounded solid is at rest. For $\tau>0$, however, a surface $\Re$ expands from point $x=0$ at a subsonic rate, that is, for a given $\tau>0$ all parts of its boundary contour $C$ lie within the volumes defined by the fronts of body waves radiating from source point $\boldsymbol{x}=0$. Unit vector $\boldsymbol{n}(\boldsymbol{x})$ defines the normal to $\Re$ and is a continuous function of $\boldsymbol{x} \in \Re$. Surface $\Re$ remains simply connected, and contour $C$ is continuous and piecewise smooth. The surface exhibits a dislocation distribution described by a jump in displacement $[\boldsymbol{u}(\boldsymbol{x}, \tau)]$ as $\Re$ is crossed in the direction of $\boldsymbol{n}$. Discontinuity $[\boldsymbol{u}]$ is finite and continuous in $(\boldsymbol{x}, \tau)$ for $\boldsymbol{x} \in \mathfrak{R}$ and finite $\tau>0$. Thus, for $\tau<0$ initial conditions are $(\boldsymbol{u}, \nabla \boldsymbol{u}) \equiv 0$, and for $\tau>0$ the linear momentum balance can, in view of (1), be written as

$$
\nabla_{k l} \epsilon_{l}=\mu \partial^{2} u_{k}+Q_{k}(\boldsymbol{x}, \tau) .
$$

Here $k=(1,2,3), l=(1,2,3,4,5,6)$, and the summation condition applies. Operator $\partial$ signifies differentiation with respect to $\tau$, and $\nabla_{k l}$ are spatial derivative operators:

$$
\left[\begin{array}{l}
\nabla_{1 l} \\
\nabla_{2 l} \\
\nabla_{3 l}
\end{array}\right]=\left[\begin{array}{lll}
C_{l 1} & C_{l 6} & C_{l 5} \\
C_{l 6} & C_{l 2} & C_{l 4} \\
C_{l 5} & C_{l 4} & C_{l 3}
\end{array}\right]\left[\begin{array}{l}
\partial_{1} \\
\partial_{2} \\
\partial_{3}
\end{array}\right], \quad C_{k l}=C_{l k} .
$$

Function $Q_{k}$ is the dislocation body-force equivalent of [Burridge and Knopoff 1964] for the homogeneous case. Discontinuity $[\boldsymbol{u}]$ is prescribed, but vector decomposition gives

$$
\begin{gathered}
{[\boldsymbol{u}]=\left[u_{N}\right] \boldsymbol{n}+\left[u_{S}\right] \boldsymbol{s},} \\
{\left[u_{N}\right]=[\boldsymbol{u}] \cdot \boldsymbol{n}, \quad\left[u_{S}\right]=|[\boldsymbol{u}]-([\boldsymbol{u}] \cdot \boldsymbol{n}) \boldsymbol{n}|,} \\
\boldsymbol{s}=\frac{[\boldsymbol{u}]-([\boldsymbol{u}] \cdot \boldsymbol{n}) \boldsymbol{n}}{|[\boldsymbol{u}]-([\boldsymbol{u}] \cdot \boldsymbol{n}) \boldsymbol{n}|}, \quad \boldsymbol{n} \cdot \boldsymbol{s}=0 .
\end{gathered}
$$

Here $\boldsymbol{s}(\boldsymbol{x}, \tau)$ is a unit vector in the plane of $\Re$. Thus,

$$
\begin{aligned}
\frac{1}{\mu} Q_{i}(\boldsymbol{x}, \tau) & =\iint_{\Re} d A G_{i k}\left(\boldsymbol{x}^{\prime}, \tau\right) \delta_{k}\left(\boldsymbol{x} ; \boldsymbol{x}^{\prime}\right), \\
G_{i k}\left(\boldsymbol{x}^{\prime}, \tau\right) & =d_{i k}^{N S}\left(\boldsymbol{x}^{\prime}, \tau\right)\left[u_{S}\left(\boldsymbol{x}^{\prime}, \tau\right)\right]+d_{i k}^{N N}\left(\boldsymbol{x}^{\prime}, \tau\right)\left[u_{N}\left(\boldsymbol{x}^{\prime}, \tau\right)\right], \\
\delta_{k}\left(\boldsymbol{x} ; \boldsymbol{x}^{\prime}\right) & =\partial_{k} \delta\left(x_{1}-x_{1}^{\prime}\right) \delta\left(x_{2}-x_{2}^{\prime}\right) \delta\left(x_{3}-x_{3}^{\prime}\right) .
\end{aligned}
$$

Area integration is over surface $\Re$ with respect to the Cartesian variable $\boldsymbol{x}^{\prime}$, and the $\delta$ symbol in (9c) is the Dirac function. Expressions for dimensionless coefficient arrays $\left(d_{i k}^{N S}, d_{i k}^{N N}\right)$ are given in terms of 
(8) in Appendix A. Combining (1)-(3) and (5)-(9) gives the uncoupled equations for $u_{j}$ :

$$
D u_{j}=D_{j k} Q_{k}(\boldsymbol{x}, \tau) .
$$

In $(9)(j, k)=(1,2,3)$, the summation convention applies, and

$$
\begin{aligned}
D & =\left(P_{11}-\partial^{2}\right)\left(P_{22}-\partial^{2}\right)\left(P_{33}-\partial^{2}\right)+2 P_{12} P_{23} P_{31}-P_{23}^{2}\left(P_{11}-\partial^{2}\right)-P_{31}^{2}\left(P_{22}-\partial^{2}\right)-P_{12}^{2}\left(P_{33}-\partial^{2}\right), \\
D_{i i} & =\left(P_{j j}-\partial^{2}\right)\left(P_{k k}-\partial^{2}\right)-P_{j k}^{2}, \\
D_{i j} & =D_{j i}=P_{i k} P_{j k}-P_{i j}\left(P_{k k}-\partial^{2}\right) .
\end{aligned}
$$

In (11) the subscripts $(i, j, k)$ are not equal and take on values $(1,2,3)$, and the $P$ terms are operators, where $P_{k l}=P_{l k}$ and

$$
\begin{aligned}
& {\left[\begin{array}{l}
P_{11} \\
P_{22} \\
P_{33}
\end{array}\right]=\boldsymbol{K}\left[\begin{array}{l}
\partial_{1}^{2} \\
\partial_{2}^{2} \\
\partial_{3}^{2}
\end{array}\right]+2 \boldsymbol{L}^{T}\left[\begin{array}{l}
\partial_{1} \partial_{2} \\
\partial_{2} \partial_{3} \\
\partial_{3} \partial_{1}
\end{array}\right],} \\
& {\left[\begin{array}{l}
P_{12} \\
P_{23} \\
P_{31}
\end{array}\right]=\boldsymbol{L}\left[\begin{array}{l}
\partial_{1}^{2} \\
\partial_{2}^{2} \\
\partial_{3}^{2}
\end{array}\right]+\boldsymbol{M}\left[\begin{array}{l}
\partial_{1} \partial_{2} \\
\partial_{2} \partial_{3} \\
\partial_{3} \partial_{1}
\end{array}\right] .}
\end{aligned}
$$

In (12) the matrices $(\boldsymbol{K}, \boldsymbol{L}, \boldsymbol{M})$ are given — see [Ting 1996] — by

$$
\begin{aligned}
\boldsymbol{K} & =\left[\begin{array}{lll}
d_{11} & d_{66} & d_{55} \\
d_{66} & d_{22} & d_{44} \\
d_{55} & d_{44} & d_{33}
\end{array}\right], \quad \boldsymbol{L}=\left[\begin{array}{lll}
d_{16} & d_{26} & d_{45} \\
d_{56} & d_{24} & d_{34} \\
d_{15} & d_{46} & d_{35}
\end{array}\right], \\
\boldsymbol{M} & =\left[\begin{array}{lll}
d_{12}+d_{66} & d_{46}+d_{25} & d_{14}+d_{56} \\
d_{46}+d_{25} & d_{23}+d_{44} & d_{45}+d_{36} \\
d_{14}+d_{56} & d_{45}+d_{36} & d_{13}+d_{55}
\end{array}\right], \\
d_{i k} & =\frac{C_{i k}}{\mu}=\frac{C_{k i}}{\mu} .
\end{aligned}
$$

It is noted that $\boldsymbol{L}$ is asymmetric. In addition $\boldsymbol{u}$ is finite as $|\boldsymbol{x}| \rightarrow \infty$ for finite $\tau>0$.

\section{Transform solution}

To determine $\boldsymbol{u}(\boldsymbol{x}, \tau)$ the unilateral Laplace transform and multiple bilateral transform are employed [van der Pol and Bremmer 1950; Sneddon 1972]:

$$
\begin{aligned}
\hat{f}(p) & =\int f(\tau) \exp (-p \tau) d \tau, \\
f^{*}\left(p, q_{1}, q_{2}, q_{3}\right) & =\iiint \hat{f}(p, \boldsymbol{x}) \exp \left[-p\left(q_{1} x_{1}+q_{2} x_{2}+q_{3} x_{3}\right)\right] d x_{1} d x_{2} d x_{3} .
\end{aligned}
$$

Here $\operatorname{Re}(p)>0$, the integration in (14a) is over positive $\tau$, and the integration in (14b) is over the entire $\operatorname{Re}\left(x_{k}\right)$-axis. Application of (14) to (6) and (9) and imposing conditions for $\tau<0$ and $|\boldsymbol{x}| \rightarrow \infty$ gives 
the formal transform solution

$$
u_{j}^{*}=\frac{q_{l}}{p} \int_{0}^{\infty} d \tau^{\prime} \iint_{\Re} d A \frac{D_{j k}}{D} G_{k l}\left(\boldsymbol{x}^{\prime}, \tau^{\prime}\right) \exp (-p)\left(q_{1} x_{1}^{\prime}+q_{2} x_{2}^{\prime}+q_{3} x_{3}^{\prime}+\tau^{\prime}\right) .
$$

In $(15)(j, k, l)=(1,2,3)$, the summation convention applies, and, from (11):

$$
\begin{aligned}
D & =\left(P_{11}-1\right)\left(P_{22}-1\right)\left(P_{33}-1\right)+2 P_{12} P_{23} P_{31}-P_{23}^{2}\left(P_{11}-1\right)-P_{31}^{2}\left(P_{22}-1\right)-P_{12}^{2}\left(P_{33}-1\right), \\
D_{i i} & =\left(P_{j j}-1\right)\left(P_{k k}-1\right)-P_{j k}^{2}, \\
D_{i j} & =D_{j i}=P_{i k} P_{j k}-P_{i j}\left(P_{k k}-1\right) .
\end{aligned}
$$

In (16) the $P$ terms follow from (12) as functions of $q_{k}$, where $P_{k l}=P_{l k}$ :

$$
\begin{aligned}
& {\left[\begin{array}{l}
P_{11} \\
P_{22} \\
P_{33}
\end{array}\right]=\boldsymbol{K}\left[\begin{array}{l}
q_{1}^{2} \\
q_{2}^{2} \\
q_{3}^{2}
\end{array}\right]+2 \boldsymbol{L}^{T}\left[\begin{array}{l}
q_{1} q_{2} \\
q_{2} q_{3} \\
q_{3} q_{1}
\end{array}\right],} \\
& {\left[\begin{array}{l}
P_{12} \\
P_{23} \\
P_{31}
\end{array}\right]=\boldsymbol{L}\left[\begin{array}{l}
q_{1}^{2} \\
q_{2}^{2} \\
q_{3}^{2}
\end{array}\right]+\boldsymbol{M}\left[\begin{array}{l}
q_{1} q_{2} \\
q_{2} q_{3} \\
q_{3} q_{1}
\end{array}\right] .}
\end{aligned}
$$

\section{Transform inversion}

The formal inversion of (14b) is [van der Pol and Bremmer 1950; Sneddon 1972]

$$
\hat{f}(p, x)=\left(\frac{p}{2 i \pi}\right)^{3} \iiint f^{*}\left(p, q_{1}, q_{2}, q_{3}\right) \exp p\left(x_{1} q_{1}+x_{2} q_{2}+x_{3} q_{3}\right) d q_{1} d q_{2} d q_{3} .
$$

Barring the existence of poles or branch points in $f^{*}$ there, integration with respect to $q_{k}$ can be taken over the entire $\operatorname{Im}\left(q_{k}\right)$-axis. Consistent with an operation commonly used in equilibrium analysis, for example, in [Ting 1996], it is convenient to introduce an orthogonal transformation

$$
\left[\begin{array}{l}
x_{1} \\
x_{2} \\
x_{3}
\end{array}\right]=\left[\begin{array}{ccc}
\cos \psi^{\prime} \sin \phi^{\prime} & \cos \psi^{\prime} \cos \phi^{\prime} & -\sin \psi^{\prime} \\
\sin \psi^{\prime} \sin \phi^{\prime} & \sin \psi^{\prime} \cos \phi^{\prime} & \cos \psi^{\prime} \\
\cos \phi^{\prime} & -\sin \phi^{\prime} & 0
\end{array}\right]\left[\begin{array}{l}
x \\
y \\
z
\end{array}\right] .
$$

Here $|x, y, z|<\infty,\left|\psi^{\prime}\right| \leq \pi / 2$, and $0 \leq \phi^{\prime} \leq \pi / 2$ so that $\left(x, \psi^{\prime}, \phi^{\prime}\right)$ represents a quasispherical coordinate system, where $\psi^{\prime}$ and $\phi^{\prime}$ correspond to the polar and azimuthal angles. Equation (19) suggests in turn the transformation

$$
q_{1}=q \cos \psi^{\prime} \sin \phi^{\prime}, \quad q_{2}=q \sin \psi^{\prime} \sin \phi^{\prime}, \quad q_{3}=q \cos \phi^{\prime} .
$$

Here $|\operatorname{Im}(q)|<\infty,\left|\psi^{\prime}\right| \leq \pi / 2$, and $0 \leq \phi^{\prime} \leq \pi / 2$. Use of (15), (19), and (20) in (18) gives

$$
\hat{u}_{j}=-\frac{2 p \partial_{l}}{\pi^{2}} \int_{0}^{\infty} d \tau^{\prime} \int_{\Phi} \sin \phi^{\prime} d \phi^{\prime} \int_{\Psi} d \psi^{\prime} \iint_{\Re} G_{k l} \frac{d A}{2 i \pi} \int|q|^{2} d q \frac{\Delta_{j k}}{\Delta} \exp p\left[q\left(x-x^{\prime}\right)-\tau^{\prime}\right] .
$$

In (21), $G_{k l}=G_{k l}\left(x^{\prime}, y^{\prime}, z^{\prime}, \psi, \phi, \tau^{\prime}\right),(j, k, l)=(1,2,3)$, and the summation convention applies. Symbols $(\Phi, \Psi)$ signify integration over, respectively, ranges $\left|\psi^{\prime}\right| \leq \pi / 2$ and $0 \leq \phi^{\prime} \leq \pi / 2$, and $q$-integration 
is over the entire $\operatorname{Im}(q)$-axis. Terms $\left(\Delta, \Delta_{j k}\right)$ are

$$
\begin{aligned}
\Delta & =I I I q^{6}-I I q^{4}+I q^{2}-1, \\
\Delta_{i i} & =\left(q^{2} B_{j j}-1\right)\left(q^{2} B_{k k}-1\right)-q^{4} B_{j k}^{2}, \\
\Delta_{i j}=\Delta_{j i} & =q^{4} B_{i k} B_{j k}-q^{2} B_{i j}\left(q^{2} B_{k k}-1\right) .
\end{aligned}
$$

In (22) we have from (17)

$$
\begin{aligned}
& {\left[\begin{array}{l}
B_{11} \\
B_{22} \\
B_{33}
\end{array}\right]=\boldsymbol{K}\left[\begin{array}{c}
\cos ^{2} \psi^{\prime} \sin ^{2} \phi^{\prime} \\
\sin ^{2} \psi^{\prime} \sin ^{2} \phi^{\prime} \\
\cos ^{2} \phi^{\prime}
\end{array}\right]+2 \boldsymbol{L}^{T}\left[\begin{array}{c}
\cos \psi^{\prime} \sin \psi^{\prime} \sin ^{2} \phi^{\prime} \\
\sin \psi^{\prime} \sin \phi^{\prime} \cos \phi^{\prime} \\
\cos \psi^{\prime} \sin \phi^{\prime} \cos \phi^{\prime}
\end{array}\right],} \\
& {\left[\begin{array}{l}
B_{12} \\
B_{23} \\
B_{31}
\end{array}\right]=\boldsymbol{L}\left[\begin{array}{c}
\cos ^{2} \psi^{\prime} \sin ^{2} \phi^{\prime} \\
\sin ^{2} \psi^{\prime} \sin ^{2} \phi^{\prime} \\
\cos ^{2} \phi^{\prime}
\end{array}\right]+\boldsymbol{M}\left[\begin{array}{c}
\cos \psi^{\prime} \sin \psi^{\prime} \sin ^{2} \phi^{\prime} \\
\sin \psi^{\prime} \sin \phi^{\prime} \cos \phi^{\prime} \\
\cos \psi^{\prime} \sin \phi^{\prime} \cos \phi^{\prime}
\end{array}\right] .}
\end{aligned}
$$

These elements constitute a symmetric matrix

$$
\boldsymbol{B}=\left[\begin{array}{lll}
B_{11} & B_{12} & B_{31} \\
B_{12} & B_{22} & B_{23} \\
B_{31} & B_{23} & B_{33}
\end{array}\right] .
$$

Terms $(I, I I, I I I)$ in (22a) are the first, second, and third invariants of $\boldsymbol{B}$. In view of (20) $B_{k l}$ are generated by a rotation in terms of $\left(\psi^{\prime}, \phi^{\prime}\right)$ involving array $d_{k l}$. Condition (4) is equivalent to the requirement that the principal minors of $C_{k l}$, and therefore $d_{k l}$, are positive definite (see also [Hohn 1964; Ting 1996]). This, in turn, guarantees that the principal minors of array (24) are positive definite and (22a) has three positive real roots. Because $\Delta$ is a cubic polynomial in $q^{2}$, this is equivalent to the condition $\alpha^{3}-\beta^{2}>0$, and so the three positive real roots are given by [Abramowitz and Stegun 1972]:

$$
\begin{gathered}
q_{A}^{2}=\frac{1}{c_{A}^{2}}, \quad q_{B}^{2}=\frac{1}{c_{B}^{2}}, \quad q_{C}^{2}=\frac{1}{c_{C}^{2}} \\
c_{A}^{2}=\frac{I}{3}+2 \sqrt{\alpha} \cos \frac{\Omega}{3}, \quad\left(c_{B}^{2}, c_{C}^{2}\right)=\frac{I}{3}-2 \sqrt{\alpha} \cos \frac{1}{3}(\Omega \pm \pi), \\
\Omega=\tan ^{-1} \frac{1}{\beta} \sqrt{\alpha^{3}-\beta^{2}}, \quad \alpha=\left(\frac{I}{3}\right)^{2}-\frac{I I}{3}, \quad \beta=\frac{I I I}{3}+\frac{I}{3}\left[\left(\frac{I}{3}\right)^{2}-\frac{I I}{2}\right] .
\end{gathered}
$$

When $\alpha^{3}-\beta^{2}=0$, two of the results in (25b) are identical. In view of (25a), (22a) can then be written as

$$
\Delta=I I I\left(q^{2}-q_{A}^{2}\right)\left(q^{2}-q_{B}^{2}\right)\left(q^{2}-q_{C}^{2}\right) .
$$

Residue theory is now used for $q$-integration in (21), where it is noted that nonanalytic function $|q|$ is defined on the $\operatorname{Im}(q)$-axis. The $\Delta_{11}$ term, for example, gives

$$
\begin{aligned}
\frac{q_{A} \Delta_{11}\left(q_{A}\right)}{2 I I I\left(q_{A}^{2}-q_{B}^{2}\right)\left(q_{A}^{2}-q_{C}^{2}\right)} \exp (-p)\left(q_{A}\left|x-x^{\prime}\right|\right. & \left.+\tau^{\prime}\right)+\frac{q_{B} \Delta_{11}\left(q_{B}\right)}{2 I I I\left(q_{B}^{2}-q_{C}^{2}\right)\left(q_{B}^{2}-q_{A}^{2}\right)} \exp (-p)\left(q_{B}\left|x-x^{\prime}\right|+\tau^{\prime}\right) \\
& +\frac{q_{C} \Delta_{11}\left(q_{C}\right)}{2 I I I\left(q_{C}^{2}-q_{A}^{2}\right)\left(q_{C}^{2}-q_{B}^{2}\right)} \exp (-p)\left(q_{C}\left|x-x^{\prime}\right|+\tau^{\prime}\right) .
\end{aligned}
$$


The exponential argument in (27) implies the existence of three wave speeds

$$
v_{A}=c_{A}\left(\psi^{\prime}, \phi^{\prime}\right) v_{S}, \quad v_{B}=c_{B}\left(\psi^{\prime}, \phi^{\prime}\right) v_{S}, \quad v_{C}=c_{C}\left(\psi^{\prime}, \phi^{\prime}\right) v_{S}
$$

Each exponential in (27) is the unilateral transform (14a) of the Dirac function [Sneddon 1972], and analogous results hold for the other $\Delta$ terms defined by (22b) and (22c). Thus the inverse of (21) is obtained by inspection:

$$
\begin{aligned}
& u_{j}=-\frac{1}{\pi^{2}} \int_{\Phi} \sin \phi^{\prime} d \phi^{\prime} \int_{\Psi} d \psi^{\prime} \frac{\partial_{l} \partial}{I I I} \int_{0}^{\tau} d \tau^{\prime} \iint_{\Re} d A \Delta_{j k}^{\prime} G_{k l}\left(T_{A}+T_{B}+T_{C}\right), \\
& T_{L}=\frac{\tau-\tau^{\prime}}{\left|x-x^{\prime}\right| M_{L}}\left(\tau-\tau^{\prime}>\frac{\left|x-x^{\prime}\right|}{c_{L}}\right) .
\end{aligned}
$$

In (29b) subscript $L$ represents $A, B$, or $C$, and

$$
\begin{aligned}
& M_{A}=\left[\left(\tau-\tau^{\prime}\right)^{2}-\left(\frac{x-x^{\prime}}{c_{B}}\right)^{2}\right]\left[\left(\tau-\tau^{\prime}\right)^{2}-\left(\frac{x-x^{\prime}}{c_{C}}\right)^{2}\right], \\
& M_{B}=\left[\left(\tau-\tau^{\prime}\right)^{2}-\left(\frac{x-x^{\prime}}{c_{C}}\right)^{2}\right]\left[\left(\tau-\tau^{\prime}\right)^{2}-\left(\frac{x-x^{\prime}}{c_{A}}\right)^{2}\right], \\
& M_{C}=\left[\left(\tau-\tau^{\prime}\right)^{2}-\left(\frac{x-x^{\prime}}{c_{A}}\right)^{2}\right]\left[\left(\tau-\tau^{\prime}\right)^{2}-\left(\frac{x-x^{\prime}}{c_{B}}\right)^{2}\right] .
\end{aligned}
$$

Function $\Delta_{j k}^{\prime}$ follows from (22b) and (22c) as

$$
\begin{aligned}
& \Delta_{i i}^{\prime}=\left[B_{j j}\left(\tau-\tau^{\prime}\right)^{2}-\left(x-x^{\prime}\right)^{2}\right]\left[B_{k k}\left(\tau-\tau^{\prime}\right)^{2}-\left(x-x^{\prime}\right)^{2}\right]-B_{j k}^{2}\left(\tau-\tau^{\prime}\right)^{4}, \\
& \Delta_{i j}^{\prime}=\Delta_{j i}^{\prime}=\left(\tau-\tau^{\prime}\right)^{2}\left(B_{i k} B_{j k}\left(\tau-\tau^{\prime}\right)^{2}-B_{i j}\left[B_{k k}\left(\tau-\tau^{\prime}\right)^{2}-\left(x-x^{\prime}\right)^{2}\right]\right) .
\end{aligned}
$$

In view of (19)

$$
x-x^{\prime}=\left(x_{1}-x_{1}^{\prime}\right) \cos \psi^{\prime} \sin \phi^{\prime}+\left(x_{2}-x_{2}^{\prime}\right) \sin \psi^{\prime} \sin \phi^{\prime}+\left(x_{3}-x_{3}^{\prime}\right) \cos \phi^{\prime} .
$$

The subsonic restriction on the expansion of $\Re$ guarantees that no part of its boundary $C$ at a given $0<\tau^{\prime}<\tau$ violates the inequality in (29b).

\section{Planar $\Re$ : Adoption of local description}

While the decomposition (8a) and (9b) allows identification of climb and glide, development of (29a) depends on function $[\boldsymbol{u}(\boldsymbol{x}, \tau)]$, and use of (9b) involves time-dependent unit vector $\boldsymbol{s}$. If a local description of displacement discontinuity, that is, in terms of the geometry of $\Re$, is available, then a more explicit result is possible — especially in the case of planar $\Re$. Then (8a) and (9b) are replaced by

$$
\begin{gathered}
{[\boldsymbol{u}]=\left[u_{T}\right] \boldsymbol{t}+\left[u_{S}\right] \boldsymbol{s}+\left[u_{N}\right] \boldsymbol{n},} \\
G_{i k}=G_{i k}(t, s, \tau)=d_{i k}^{N T}\left[u_{T}\right]+d_{i k}^{N S}\left[u_{S}\right]+d_{i k}^{N N}\left[u_{N}\right] .
\end{gathered}
$$


Unit vectors $(\boldsymbol{t}, \boldsymbol{s}, \boldsymbol{n})$ form a fixed right-handed set $(\boldsymbol{t} \times \boldsymbol{s}=\boldsymbol{n}, \boldsymbol{s} \times \boldsymbol{n}=\boldsymbol{t}, \boldsymbol{n} \times \boldsymbol{t}=\boldsymbol{s})$. They are invariant in $\Re$, but the $(T, S, N)$ components of $\boldsymbol{u}$ are functions of $(t, s, \tau)$, where $(t, s)$ correspond to $(\boldsymbol{t}, \boldsymbol{s})$ and

$$
\left[\begin{array}{l}
\boldsymbol{n} \\
\boldsymbol{t} \\
\boldsymbol{s}
\end{array}\right]=\left[\begin{array}{lll}
n_{1} & n_{2} & n_{3} \\
t_{1} & t_{2} & t_{3} \\
s_{1} & s_{2} & s_{3}
\end{array}\right]\left[\begin{array}{l}
\boldsymbol{e}_{1} \\
\boldsymbol{e}_{2} \\
\boldsymbol{e}_{3}
\end{array}\right] .
$$

Terms $\left(n_{k}, t_{k}, s_{k}\right)$ are (constant) direction cosines, $\boldsymbol{e}_{k}$ are Cartesian basis vectors, and dimensionless coefficient arrays $\left(d_{i k}^{N T}, d_{i k}^{N S}, d_{i k}^{N N}\right)$ are given in Appendix B. Normal $\boldsymbol{n}$ is known, but vectors $(\boldsymbol{t}, \boldsymbol{s})$ in the plane of $\Re$ are somewhat arbitrary. In light of (19), a convenient choice is to define $\boldsymbol{n}$ in terms of polar and azimuthal angles $(\psi, \phi)$, where $|\psi|<\pi / 2$ and $0<\phi<\pi / 2$, and introduce for integration over $\mathfrak{R}$ the transformation and corresponding direction cosines:

$$
\begin{aligned}
& {\left[\begin{array}{l}
x_{1}^{\prime} \\
x_{2}^{\prime} \\
x_{3}^{\prime}
\end{array}\right]=\left[\begin{array}{ccc}
\cos \psi \sin \phi & \cos \psi \cos \phi & -\sin \psi \\
\sin \psi \sin \phi & \sin \psi \cos \phi & \cos \psi \\
\cos \phi & -\sin \phi & 0
\end{array}\right]\left[\begin{array}{l}
0 \\
t \\
s
\end{array}\right],} \\
& n_{1}=\cos \psi \sin \phi, \quad n_{2}=\sin \psi \sin \phi, \quad n_{3}=\cos \phi, \\
& t_{1}=\cos \psi \cos \phi, \quad t_{2}=-\sin \psi \cos \phi, \quad t_{3}=-\sin \phi, \\
& s_{1}=-\sin \psi, \quad s_{2}=\cos \psi, \quad s_{3}=0 .
\end{aligned}
$$

In view of (33)-(35) the integrand of the $\psi^{\prime} \phi^{\prime}$-integration in (29a) can be written as

$$
\begin{aligned}
d_{k l}^{N T} \partial \int_{0}^{\tau} d \tau^{\prime} \iint_{\Re} d A\left[P_{j k} \partial_{l}^{\prime}\left[u_{T}\right]-\partial_{l}^{\prime}\left(P_{j k}\left[u_{T}\right]\right)\right] & +d_{k l}^{N S} \partial \int_{0}^{\tau} d \tau^{\prime} \iint_{\Re} d A\left[P_{j k} \partial_{l}^{\prime}\left[u_{S}\right]-\partial_{l}^{\prime}\left(P_{j k}\left[u_{S}\right]\right)\right] \\
& +d_{k l}^{N N} \partial \int_{0}^{\tau} d \tau^{\prime} \iint_{\Re} d A\left[P_{j k} \partial_{l}^{\prime}\left[u_{N}\right]-\partial_{l}^{\prime}\left(P_{j k}\left[u_{N}\right]\right)\right],
\end{aligned}
$$

where

$$
P_{j k}=\Delta_{j k}^{\prime}\left(T_{A}+T_{B}+T_{C}\right)
$$

Here $\partial_{l}^{\prime}$ represents differentiation with respect to $x_{l}^{\prime}$ in (32), and $\left(d_{k l}^{N T}, d_{k l}^{N S}, d_{k l}^{N}\right)$ are independent of $\left(t, s, \tau^{\prime}\right)$. From (35a), $P_{j k}$ and $\left(\left[u_{T}\right],\left[u_{S}\right],\left[u_{N}\right]\right)$ depend on $\left(t, s, \tau^{\prime}\right)$ and

$$
\partial_{1}^{\prime} \rightarrow \cos \psi \sin \phi \frac{\partial}{\partial t}-\sin \psi \frac{\partial}{\partial s}, \quad \partial_{2}^{\prime} \rightarrow \sin \psi \cos \phi \frac{\partial}{\partial t}+\cos \psi \frac{\partial}{\partial s}, \quad \partial_{3}^{\prime} \rightarrow \sin \phi \frac{\partial}{\partial t} .
$$

Use of (37) in (36) and application of Stokes's theorem [Hay 1953] allows the second terms in (36) to be replaced by line integrals around contour $C$. For example, the first integral over $\Re$ in (36) gives for $l=(1,2,3)$, respectively,

$$
\begin{gathered}
\iint_{\Re} d A P_{j k}\left(\cos \psi \sin \phi \frac{\partial}{\partial t}-\sin \psi \frac{\partial}{\partial s}\right)\left[u_{T}\right]+\oint_{C} d c P_{j k}\left[u_{T}\right](\sin \psi \boldsymbol{t}+\cos \psi \sin \phi s) \cdot \boldsymbol{t}_{C} \\
\iint_{\Re} d A P_{j k}\left(\sin \psi \cos \phi \frac{\partial}{\partial t}+\cos \psi \frac{\partial}{\partial s}\right)\left[u_{T}\right]-\oint_{C} d c P_{j k}\left[u_{T}\right](\cos \psi \boldsymbol{t}-\sin \psi \cos \phi s) \cdot \boldsymbol{t}_{C}, \\
\sin \phi \iint_{\Re} d A P_{j k} \frac{\partial}{\partial t}\left[u_{T}\right]+\sin \phi \oint_{C} d c P_{j k}\left[u_{T}\right] \boldsymbol{s} \cdot \boldsymbol{t}_{C} .
\end{gathered}
$$


Integration is counterclockwise around $C$ in the $t s$-plane, $t_{C}$ is the unit vector tangent to $C$ and in the direction of integration, and (32) takes the form

$x-x^{\prime}=\left(x_{1} \cos \psi^{\prime}+x_{2} \sin \psi^{\prime}\right) \sin \phi^{\prime}+x_{3} \cos \phi^{\prime}$

$$
-\left[\sin \phi^{\prime} \cos \phi \cos \left(\psi^{\prime}-\psi\right)-\cos \phi^{\prime} \sin \phi\right] t+\sin \phi^{\prime} \sin \left(\psi^{\prime}-\psi\right) s .
$$

The subsonic restriction on the growth of $\Re$ guarantees that the wave front history defined in (29b) does not affect the limits of integration over $(\Re, C)$.

\section{Planar $\mathfrak{R}$ : Traction field}

A local description does not imply knowledge of the traction field $\left(\sigma_{N S}, \sigma_{N T}, \sigma_{N N}\right)$ on $\Re$. However, with (29) and (36)-(39) in hand, this field can be obtained by evaluation of the following expressions for $\boldsymbol{x} \in \mathfrak{R}:$

$$
\begin{aligned}
& {\left[\begin{array}{c}
\sigma_{N N} \\
\sigma_{T T} \\
\sigma_{S S} \\
\sigma_{S N} \\
\sigma_{N T} \\
\sigma_{T S}
\end{array}\right]=\mu\left[\begin{array}{llllll}
d_{11}^{N N} & d_{22}^{N N} & d_{33}^{N N} & d_{23}^{N N} & d_{31}^{N N} & d_{12}^{N N} \\
d_{11}^{T T} & d_{22}^{T T} & d_{33}^{T T} & d_{23}^{T T} & d_{31}^{T T} & d_{12}^{T T} \\
d_{11}^{S S} & d_{22}^{S S} & d_{33}^{S S} & d_{23}^{S S} & d_{31}^{S S} & d_{12}^{S S} \\
d_{11}^{S N} & d_{22}^{S N} & d_{33}^{S N} & d_{23}^{S N} & d_{31}^{S N} & d_{12}^{S N} \\
d_{11}^{N T} & d_{22}^{N T} & d_{33}^{N T} & d_{23}^{N T} & d_{31}^{N T} & d_{12}^{N T} \\
d_{11}^{T S} & d_{22}^{T S} & d_{33}^{T S} & d_{23}^{T S} & d_{31}^{T S} & d_{12}^{T S}
\end{array}\right]\left[\begin{array}{c}
\partial_{1} u_{1} \\
\partial_{2} u_{2} \\
\partial_{3} u_{3} \\
\partial_{2} u_{3}+\partial_{3} u_{2} \\
\partial_{3} u_{1}+\partial_{1} u_{3} \\
\partial_{1} u_{2}+\partial_{2} u_{1}
\end{array}\right],} \\
& d_{i k}^{S N}=d_{i k}^{N S}, \quad d_{i k}^{N T}=d_{i k}^{T N}, \quad d_{i k}^{T S}=d_{i k}^{S T} .
\end{aligned}
$$

Dimensionless coefficient arrays $\left(d_{i k}^{N S}, d_{i k}^{N T}, d_{i k}^{N N}\right)$ appear in (33), and are defined in Appendix B. Definitions of coefficient arrays $\left(d_{i k}^{T T}, d_{i k}^{S S}, d_{i k}^{T S}\right)$ are also found there.

\section{Planar $\Re$ : Two special cases with application}

Dip-slip and strike-slip faulting in seismology [Canitez and Toksoz 1972] and slip mechanisms in a crystal lattice [Read 1953] can be modeled as a spatially invariant dislocation distribution on an expanding surface. For such a distribution on $\Re$, the terms in $(38)$ reduce to

$$
\begin{gathered}
{\left[u_{T}\right] \oint_{C} d c P_{j k}(\sin \psi t+\cos \psi \sin \phi \boldsymbol{s}) \cdot \boldsymbol{t}_{C},} \\
-\left[u_{T}\right] \oint_{C} d c P_{j k}(\cos \psi \boldsymbol{t}-\sin \psi \cos \phi \boldsymbol{s}) \cdot \boldsymbol{t}_{C}, \\
{\left[u_{T}\right] \sin \phi \oint_{C} d c P_{j k} \boldsymbol{s} \cdot \boldsymbol{t}_{C} .}
\end{gathered}
$$

The use of dislocation distributions that exhibit spatial variation to model internal cracks is well established [Bilby and Eshelby 1968; Barber 1992]. If $\Re$ represents a crack plane, then $[\boldsymbol{u}]=0(\boldsymbol{x} \in C)$ and only the integration over $\Re$ in (38) remains. Results analogous to (41) exist, for example, [Brock 1986], for a nonplanar surface in an isotropic solid. 
If the additional condition is imposed that a radial line from $\boldsymbol{x}=0$ to any point on $C$ lies within $\Re$, it is convenient to define $(\Re, C)$ in terms of polar coordinates:

$$
\begin{gathered}
\Re: r<r_{C}\left(\theta^{\prime}, \tau\right), \quad C: r=r_{C}\left(\theta^{\prime}, \tau\right), \\
t=r \cos \theta^{\prime}, \quad s=r \sin \theta^{\prime} \quad\left(0 \leq \theta^{\prime} \leq 2 \pi\right) .
\end{gathered}
$$

Here $\left(r_{C}, \partial r_{C} / \partial \theta^{\prime}\right)$ are single-valued and continuous in $\theta^{\prime}$. Quantities $\left(\partial r_{C} / \partial \theta^{\prime}, \partial r_{C}\right)$ are finite and, in particular, $\partial r_{C}<\min \left[c_{A}(\psi, \phi), c_{B}(\psi, \phi), c_{C}(\psi, \phi)\right]$. Use of (42) in (41c), for example, gives

$$
\left[u_{T}\right] \sin \phi \int_{0}^{2 \pi} P_{j k} \frac{\partial}{\partial \theta^{\prime}}\left(r_{C} \sin \theta^{\prime}\right) d \theta^{\prime} .
$$

For the crack plane case, the corresponding result is

$$
\sin \phi \int_{0}^{2 \pi} P_{j k} d \theta^{\prime} \int_{0}^{r_{C}} d r\left(r \frac{\partial}{\partial r} \sin \theta^{\prime}+\cos \theta^{\prime} \frac{\partial}{\partial \theta^{\prime}}\right)\left[u_{T}\right] .
$$

In the case of (44),

$$
\begin{aligned}
x-x^{\prime} & =\left(x_{1} \cos \psi^{\prime}+x_{2} \sin \psi^{\prime}\right) \sin \phi^{\prime}+x_{3} \cos \phi^{\prime}-r F\left(\phi, \phi^{\prime}, \psi^{\prime}-\psi, \theta^{\prime}\right), \\
F & =\left[\cos \theta^{\prime} \sin \left(\psi^{\prime}-\psi\right)+\cos \phi \sin \theta^{\prime} \cos \left(\psi^{\prime}-\psi\right)\right] \sin \phi^{\prime}-\sin \theta^{\prime} \sin \phi \cos \phi^{\prime} .
\end{aligned}
$$

For (43), the symbol $r$ in (45a) is replaced with $r_{C}\left(\theta^{\prime}, \tau^{\prime}\right)$.

\section{Limit results}

For an orthotropic solid [Ting 1996; Jones 1999] matrix $\boldsymbol{K}$ is again defined by (13a), but $\boldsymbol{L}=0$ and

$$
\boldsymbol{M}=\left[\begin{array}{ccc}
d_{12}+d_{66} & 0 & 0 \\
0 & d_{23}+d_{44} & 0 \\
0 & 0 & d_{13}+d_{55}
\end{array}\right]
$$

For transverse isotropy with respect to the $x_{1} x_{2}$-plane [Ting 1996; Jones 1999] $\boldsymbol{L}=0$ and

$$
\begin{aligned}
\boldsymbol{K} & =\left[\begin{array}{lll}
d_{11} & d_{66} & d_{55} \\
d_{66} & d_{11} & d_{55} \\
d_{55} & d_{55} & d_{33}
\end{array}\right], \\
\boldsymbol{M} & =\left[\begin{array}{ccc}
d_{11}-d_{66} & 0 & 0 \\
0 & d_{13}+d_{55} & 0 \\
0 & 0 & d_{13}+d_{55}
\end{array}\right] .
\end{aligned}
$$

For a cubic solid [Crandall and Dahl 1959] $\boldsymbol{L}=0$ and

$$
\boldsymbol{K}=\left[\begin{array}{ccc}
d_{11} & 1 & 1 \\
1 & d_{11} & 1 \\
1 & 1 & d_{11}
\end{array}\right], \quad \boldsymbol{M}=\left(d_{12}+1\right) \mathbf{1}
$$

Here $\mathbf{1}$ is the identity tensor. For isotropy [Ting 1996; Jones 1999] $\boldsymbol{L}=0, \boldsymbol{K}$ is defined by (48), and

$$
\boldsymbol{M}=\left(d_{11}-1\right) \mathbf{1} .
$$


Equations (25), (29), and (30) still hold for the orthotropic solid. Transverse isotropy (47) yields the more explicit results

$$
\begin{aligned}
c_{A} & =\sqrt{d_{44} \cos ^{2} \phi^{\prime}+}+d_{66} \sin ^{2} \phi^{\prime} \\
\left(c_{A}^{2}, c_{C}^{2}\right) & =\frac{1}{2}\left(d_{44}+d_{11} \sin ^{2} \phi^{\prime}+d_{33} \cos ^{2} \phi^{\prime}\right) \\
& \pm \frac{1}{2} \sqrt{\left(d_{33} \cos ^{2} \phi^{\prime}-d_{11} \sin ^{2} \phi^{\prime}-d_{4} \cos 2 \phi^{\prime}\right)^{2}+\left(d_{44}+d_{13}\right)^{2} \sin ^{2} 2 \phi^{\prime}} .
\end{aligned}
$$

Moreover, $T_{B}+T_{C}$ replaces $T_{A}+T_{B}+T_{C}$ in terms that involve $\left(d_{31}^{N T}, d_{31}^{N S}, d_{31}^{N N}\right)$ and $\left(d_{23}^{N T}, d_{23}^{N S}, d_{23}^{N N}\right)$ in (37), where

$$
\begin{aligned}
T_{L} & =\frac{\tau-\tau^{\prime}}{M_{L}\left|x-x^{\prime}\right|} \quad\left(\tau-\tau^{\prime}>\frac{\left|x-x^{\prime}\right|}{c_{L}}\right), \\
M_{B} & =\left(\tau-\tau^{\prime}\right)^{2}-\left(\frac{x-x^{\prime}}{c_{C}}\right)^{2}, \quad M_{C}=\left(\tau-\tau^{\prime}\right)^{2}-\left(\frac{x-x^{\prime}}{c_{B}}\right)^{2} .
\end{aligned}
$$

It should be noted that conditions specific to transversely isotropic solids that guarantee that $\alpha^{3}-\beta^{2}>0$ are given in detail in [Payton 1983]. For the cubic solid

$$
\begin{aligned}
c_{A} & =1, \\
\left(c_{B}^{2}, c_{C}^{2}\right) & =\frac{1}{2}\left(d_{11}+1\right) \pm \frac{1}{2} \sin \phi^{\prime} \sqrt{\left(d_{11}-1\right)^{2} \sin ^{2} \phi^{\prime}+\left(d_{12}+1\right)^{2} \cos ^{2} \phi^{\prime}} .
\end{aligned}
$$

The results associated with (51) still hold. For the isotropic solid $c_{A}=c_{C}$, so only two speeds $\left(v_{B}, v_{C}\right)=$ $\left(c_{B}, c_{C}\right) v_{S}$ exist, where

$$
c_{B}=\sqrt{d_{11}}, \quad c_{C}=1 \text {. }
$$

Thus, isotropy corresponds to the case $\alpha^{3}-\beta^{2}=0$ noted above, and (36) involves only terms of the type (51).

As an illustration of wave speed variation with propagation direction, a transversely isotropic graphiteepoxy [Jones 1999] is considered with

$$
\begin{aligned}
\mu=C_{44}= & 7.07 \mathrm{GPa}, \quad v_{S}=2546 \mathrm{~m} / \mathrm{s}, \quad d_{44}=1.0, \quad d_{66}=0.4951, \\
& {\left[\begin{array}{lll}
d_{11} & d_{12} & d_{31} \\
d_{12} & d_{11} & d_{31} \\
d_{31} & d_{31} & d_{33}
\end{array}\right]=\left[\begin{array}{lll}
1.9689 & 0.9788 & 0.9109 \\
0.9788 & 1.9689 & 0.9109 \\
0.9109 & 0.9109 & 22.73
\end{array}\right] . }
\end{aligned}
$$

Use of these properties in (50) gives the values of dimensionless speeds $\left(c_{A}, c_{B}, c_{C}\right)$ shown in Table 1 for $0 \leq \phi^{\prime} \leq 90^{\circ}$. It is seen that the values are quite sensitive to $\phi^{\prime}$; compare [Wang and Achenbach 1994].

\section{Concluding remarks}

The present formulation leads to transient expressions that are multiple integrals in a unit spherical quadrant in terms of polar and azimuthal angles, that is, $\left(\left|\psi^{\prime}\right|<\pi / 2,0 \leq \phi^{\prime} \leq \pi / 2\right)$, but are explicit functions of Cartesian principal material coordinates. Approaches used to produce the anisotropic Green's function, for example, [Wang and Achenbach 1995; Ting 1996], are similar but feature integration over the surface 


\begin{tabular}{rlcl}
\hline & \multicolumn{1}{c}{$c_{A}$} & $c_{B}$ & \multicolumn{1}{c}{$c_{C}$} \\
\hline$\phi^{\prime}=0^{\circ}$ & 1.0 & 4.8197 & 0.7071 \\
$15^{\circ}$ & 0.9829 & 4.6650 & 0.7595 \\
$30^{\circ}$ & 0.9348 & 4.2094 & 0.9057 \\
$45^{\circ}$ & 0.8646 & 3.4926 & 1.0730 \\
$60^{\circ}$ & 0.7882 & 2.5908 & 1.2029 \\
$75^{\circ}$ & 0.7272 & 1.7096 & 1.1986 \\
$90^{\circ}$ & 0.7036 & 1.4042 & 1.0 \\
\hline
\end{tabular}

Table 1. Graphite-epoxy: dimensionless speeds $\left(v_{S}=2546 \mathrm{~m} / \mathrm{s}\right)$.

of a unit sphere or around a unit circle [Synge 1957]. Nevertheless, for a planar dislocation surface, the present integration process can in fact be carried out in terms of a polar coordinate system $\left(r, \theta^{\prime}\right)$ in the plane. Moreover, Stokes's theorem [Hay 1953] can be invoked to replace portions of the area integration with integration around the area contour. The description of the dislocation distribution in terms of surface geometry does produce lengthier solution expressions. However, climb and glide mechanisms can be identified explicitly in terms of matrix/tensor arrays that are similar to those defined in analyses of general anisotropy; see [Ting 1996], for example. Related arrays that characterize the orientation of the dislocation surface and its climb and glide components with respect to the principal material axes arise, and resemble in form the constitutive relation for the solid itself. In summary, the disadvantages of the length and complexity of these solutions, and their combination of standard coordinate systems, may be compensated for by a more explicit nature. This nature may be of advantage in cases where solution response to particular dislocation features is of interest.

\section{Appendix A}

The dimensionless coefficient array $d_{i k}^{N S}\left(\boldsymbol{x}^{\prime}, \tau\right)$ is defined by

$$
\begin{gathered}
{\left[\begin{array}{l}
d_{11}^{N S} \\
d_{22}^{N S} \\
d_{33}^{N S} \\
d_{23}^{N S} \\
d_{31}^{N S} \\
d_{12}^{N S}
\end{array}\right]=\left[\begin{array}{llllll}
d_{1} & d_{12} & d_{13} & d_{14} & d_{15} & d_{16} \\
d_{12} & d_{22} & d_{23} & d_{24} & d_{25} & d_{26} \\
d_{13} & d_{23} & d_{33} & d_{34} & d_{35} & d_{36} \\
d_{14} & d_{24} & d_{34} & d_{44} & d_{45} & d_{46} \\
d_{15} & d_{25} & d_{35} & d_{45} & d_{55} & d_{56} \\
d_{16} & d_{26} & d_{36} & d_{46} & d_{56} & d_{66}
\end{array}\right]\left[\begin{array}{c}
s_{1} n_{1} \\
s_{2} n_{2} \\
s_{3} n_{3} \\
s_{2} n_{3}+s_{3} n_{2} \\
s_{3} n_{1}+s_{1} n_{3} \\
s_{1} n_{2}+s_{2} n_{1}
\end{array}\right],} \\
d_{i k}^{N S}=d_{k i}^{N S} .
\end{gathered}
$$

Here $s_{l}\left(\boldsymbol{x}^{\prime}, \tau\right)$ and $n_{l}\left(\boldsymbol{x}^{\prime}\right)$ are the direction cosines of $(\boldsymbol{s}, \boldsymbol{n})$ in the principal material basis. It is noted that (A.1) resembles constitutive equations (1)-(3), with $d_{i k}^{N S}$ playing the role of (dimensionless) stresses, and $\left(s_{i} n_{i}, s_{i} n_{k}+s_{k} n_{i}\right)$ acting as strains $\left(\partial_{i} u_{i}, \partial_{i} u_{k}+\partial_{k} u_{i}\right)$. Coefficients $d_{i k}^{N N}$ follow by replacing $s_{l}$ with $n_{l}$. 


\section{Appendix B}

Dimensionless coefficient array $d_{i k}^{N T}$ is defined by

$$
\begin{aligned}
{\left[\begin{array}{l}
d_{11}^{N T} \\
d_{22}^{N T} \\
d_{33}^{N T} \\
d_{23}^{N T} \\
d_{31}^{N T} \\
d_{12}^{N T}
\end{array}\right] } & =\left[\begin{array}{llllll}
d_{11} & d_{12} & d_{13} & d_{14} & d_{15} & d_{16} \\
d_{12} & d_{22} & d_{23} & d_{24} & d_{25} & d_{26} \\
d_{13} & d_{23} & d_{33} & d_{34} & d_{35} & d_{36} \\
d_{14} & d_{24} & d_{34} & d_{44} & d_{45} & d_{46} \\
d_{15} & d_{25} & d_{35} & d_{45} & d_{55} & d_{56} \\
d_{16} & d_{26} & d_{36} & d_{46} & d_{56} & d_{66}
\end{array}\right]\left[\begin{array}{c}
t_{1} n_{1} \\
t_{2} n_{2} \\
t_{3} n_{3} \\
t_{2} n_{3}+t_{3} n_{2} \\
t_{3} n_{1}+t_{1} n_{3} \\
t_{1} n_{2}+t_{2} n_{1}
\end{array}\right], \\
d_{i k}^{N T} & =d_{k i}^{N T} .
\end{aligned}
$$

Terms $\left(t_{l}, n_{l}\right)$ are direction cosines of $(\boldsymbol{t}, \boldsymbol{n})$ in the principal material basis. For planar $\Re$, of course, direction cosines are constant. Results for $\left(d_{i k}^{N S}, d_{i k}^{N N}\right)$ follow from (B.1) by replacing direction cosine $t_{l}$ with, respectively, direction cosine $\left(s_{l}, n_{l}\right)$.

Another set of dimensionless coefficients $\left(d_{i k}^{T S}, d_{i k}^{T T}, d_{i k}^{S S}\right)$ can also be defined, where

$$
\begin{aligned}
{\left[\begin{array}{c}
d_{11}^{T S} \\
d_{22}^{T S} \\
d_{33}^{T S} \\
d_{23}^{T S} \\
d_{31}^{T S} \\
d_{12}^{T S}
\end{array}\right] } & =\left[\begin{array}{llllll}
d_{11} & d_{12} & d_{13} & d_{14} & d_{15} & d_{16} \\
d_{12} & d_{22} & d_{23} & d_{24} & d_{25} & d_{26} \\
d_{13} & d_{23} & d_{33} & d_{34} & d_{35} & d_{36} \\
d_{14} & d_{24} & d_{34} & d_{44} & d_{45} & d_{46} \\
d_{15} & d_{25} & d_{35} & d_{45} & d_{55} & d_{56} \\
d_{16} & d_{26} & d_{36} & d_{46} & d_{56} & d_{66}
\end{array}\right]\left[\begin{array}{c}
t_{1} s_{1} \\
t_{2} s_{2} \\
t_{3} s_{3} \\
t_{2} s_{3}+t_{3} s_{2} \\
t_{3} s_{1}+t_{1} s_{3} \\
t_{1} s_{2}+t_{2} s_{1}
\end{array}\right], \\
d_{i k}^{T S} & =d_{k i}^{T S} .
\end{aligned}
$$

Terms $\left(d_{i k}^{T T}, d_{i k}^{S S}\right)$ follow from (B.2) by, respectively, replacing $s_{l}$ with $t_{l}$ and $t_{l}$ with $s_{l}$.

Equations (B.1) and (B.2) also show that

$$
d_{i k}^{T N}=d_{k i}^{N T}, \quad d_{i k}^{S N}=d_{i k}^{N S}, \quad d_{i k}^{T S}=d_{i k}^{S T} .
$$

Equations (B.1) and (B.2) resemble (A.1), so that the forms of $\left(d_{i k}^{N T}, d_{i k}^{N S}, d_{i k}^{T S}\right)$ also resemble constitutive equations (1)-(3).

\section{References}

[Abramowitz and Stegun 1972] M. Abramowitz and I. A. Stegun, Handbook of mathematical functions: With formulas, graphs, and mathematical tables, Dover, New York, 1972.

[Barber 1992] J. R. Barber, Elasticity, Solid Mechanics and its Applications 12, Kluwer, Dordrecht, 1992.

[Barnett and Lothe 1973] D. M. Barnett and J. Lothe, "Synthesis of the sextic and the integral formalism for dislocations, Green's function and surface waves in anisotropic elastic solids", Physica Norvegica 7 (1973), 13-19.

[Bilby and Eshelby 1968] B. A. Bilby and J. D. Eshelby, "Dislocations and the theory of fracture", pp. 99-182 in Fracture, vol. 1, edited by H. Liebowitz, Academic Press, New York, 1968.

[Brock 1986] L. M. Brock, "A transient three dimensional analysis of non-uniform dislocation distribution growth by climb and glide over non-planar surfaces", Proceedings of the Royal Society of London, A 407 (1986), 299-311. 
[Burridge and Knopoff 1964] R. Burridge and L. Knopoff, "Body force equivalents for seismic dislocations", Bulletin of the Seismological Society of America 54 (1964), 1875-1888.

[Canitez and Toksoz 1972] N. Canitez and M. N. Toksoz, "Static and dynamic study of earthquake source mechanisms: San Fernando earthquake", Journal of Geophysical Research 77 (1972), 2583-2594.

[Crandall and Dahl 1959] S. H. Crandall and N. C. Dahl, An introduction to the mechanics of solids, McGraw-Hill, New York, 1959.

[Hay 1953] G. E. Hay, Vector and tensor analysis, Dover, New York, 1953.

[Hohn 1964] F. E. Hohn, Elementary matrix algebra, 2nd ed., Macmillan, New York, 1964.

[Jones 1999] R. M. Jones, Mechanics of composite materials, 2nd ed., Brunner-Routledge, New York, 1999.

[Knopoff and Gilbert 1960] L. Knopoff and F. Gilbert, "First motions from seismic sources", Bulletin of the Seismological Society of America 134 (1960), 117-134.

[Nabarro 1951] F. R. N. Nabarro, "The synthesis of elastic dislocation fields", Philos. Mag. (7) 42 (1951), 1224-1231.

[Payton 1983] R. G. Payton, Elastic wave propagation in transversely isotropic media, Martinus Nijhoff, The Hague, 1983.

[van der Pol and Bremmer 1950] B. van der Pol and H. Bremmer, Operational calculus, based on the two-sided Laplace integral, Cambridge University Press, 1950.

[Read 1953] W. T. Read, Dislocations in crystals, McGraw-Hill, New York, 1953.

[Sneddon 1972] I. N. Sneddon, The use of integral transforms, McGraw-Hill, New York, 1972.

[Stroh 1958] A. N. Stroh, "Dislocations and cracks in anisotropic elasticity", Phil. Mag. (8) 3 (1958), 625-646.

[Stroh 1962] A. N. Stroh, "Steady state problems in anisotropic elasticity", J. Math. and Phys. 41 (1962), 77-103.

[Synge 1957] J. L. Synge, The hypercircle in mathematical physics: a method for the approximate solution of boundary value problems, Cambridge University Press, 1957.

[Ting 1996] T. C. T. Ting, Anisotropic elasticity: Theory and applications, Oxford Engineering Science Series 45, Oxford University Press, New York, 1996.

[Ting and Lee 1997] T. C. T. Ting and V.-G. Lee, "The three-dimensional elastostatic Green's function for general anisotropic linear elastic solids", Quart. J. Mech. Appl. Math. 50:3 (1997), 407-426.

[Wang and Achenbach 1994] C.-Y. Wang and J. D. Achenbach, "Elastodynamic fundamental solutions for anisotropic solids", Geophysics Journal International 118 (1994), 384-392.

[Wang and Achenbach 1995] C.-Y. Wang and J. D. Achenbach, "Three-dimensional time-harmonic elastodynamic Green's functions for anisotropic solids", Proc. Roy. Soc. London Ser. A 449:1937 (1995), 441-458.

[Willis 1980] J. R. Willis, "A polarization approach to the scattering of elastic waves, I: Scattering by a single inclusion", $J$. Mech. Phys. Solids 28:5-6 (1980), 287-305.

Received 7 Jan 2013. Revised 5 Mar 2013. Accepted 5 Mar 2013.

LOUIS MiLTON BROCK: brock@engr.uky.edu

Department of Mechanical Engineering, University of Kentucky, 265 Ralph G. Anderson Building, Lexington, KY 40506-0503, United States 


\title{
JOURNAL OF MECHANICS OF MATERIALS AND STRUCTURES
}

\author{
msp.org/jomms
}

\author{
Founded by Charles R. Steele and Marie-Louise Steele
}

EDITORIAL BOARD

ADAIR R. AGUIAR

KATIA BERTOLDI

University of São Paulo at São Carlos, Brazil

DAVIDE BIGONI

Harvard University, USA

IWONA JASIUK

University of Trento, Italy

Thomas J. PENCE

University of Illinois at Urbana-Champaign, USA

YASUHIDE SHINDO

Michigan State University, USA

DAVID STEIGMANN

Tohoku University, Japan

University of California at Berkeley

\section{ADVISORY BOARD}

J. P. CARTER University of Sydney, Australia

R. M. Christensen Stanford University, USA

G. M. L. GLADWELL University of Waterloo, Canada

D. H. Hodges Georgia Institute of Technology, USA

J. HUTCHINSON Harvard University, USA

C. HwU National Cheng Kung University, Taiwan

B. L. KARIHALOO University of Wales, UK

Y. Y. KIM Seoul National University, Republic of Korea

Z. Mroz Academy of Science, Poland

D. PAMPlonA Universidade Católica do Rio de Janeiro, Brazil

M. B. RubiN Technion, Haifa, Israel

A. N. SHUPIKOV Ukrainian Academy of Sciences, Ukraine

T. TARNAI University Budapest, Hungary

F. Y. M. WAN University of California, Irvine, USA

P. WRIGGERS Universität Hannover, Germany

W. YANG Tsinghua University, China

F. ZIEGLER Technische Universität Wien, Austria

PRODUCTION production@msp.org

SILVIO LEVY Scientific Editor

Cover photo: Mando Gomez, www.mandolux.com

See msp.org/jomms for submission guidelines.

JoMMS (ISSN 1559-3959) at Mathematical Sciences Publishers, 798 Evans Hall \#6840, c/o University of California, Berkeley, CA 94720-3840, is published in 10 issues a year. The subscription price for 2013 is US \$555/year for the electronic version, and $\$ 705 /$ year (+\$60, if shipping outside the US) for print and electronic. Subscriptions, requests for back issues, and changes of address should be sent to MSP.

JoMMS peer-review and production is managed by EditFLOW ${ }^{\circledR}$ from Mathematical Sciences Publishers.

PUBLISHED BY

mathematical sciences publishers

nonprofit scientific publishing

http://msp.org/

(C) 2013 Mathematical Sciences Publishers 


\section{Journal of Mechanics of Materials and Structures}

\section{Volume 8, No.:2-4 April-June 2013}

A diffuse cohesive energy approach to fracture and plasticity: the one-dimensional case

Gianpietro Del Piero, Giovanni Lancioni and Riccardo March 109

Continuum deployable shells made of thin plates

Vladimir A. GracheV and Yuriy S. NeUSTAdT

Self-folding of a slender microbeam and thin film: an elastica model

JIANLIN LIU and JUNG HoON LEE 169

Dispersion of guided waves in initially stressed layered plates

JIANGONG YU and SHENLEI LI 185

Empirical models for predicting protective properties of concrete shields against

high-speed impact

GABI BEN-Dor, ANATOLY DUBinsky and Tov ELPERIN

Transient response of a general anisotropic solid to dislocation growth: alternative formulation LOUIS MILTON BROCK 